Published in final edited form as:

J Am Chem Soc. 2019 July 24; 141(29): 11522-11530. doi:10.1021/jacs.9b02895.

\title{
Genetically Targeted Optical Control of an Endogenous G Protein-Coupled Receptor
}

\author{
Prashant C. Donthamsetti ${ }^{\dagger}$, Johannes Broichhagen ${ }^{\ddagger}$, Vojtech Vyklicky ${ }^{\dagger}$, Cherise Stanley ${ }^{\dagger}$, \\ Zhu Fu ${ }^{\dagger}$, Meike Visel ${ }^{\dagger}$, Joshua L. Levitz ${ }^{\S}$, Jonathan A. Javitch ${ }^{\|, \perp}$, Dirk Trauner ${ }^{\#}$, Ehud Y. \\ Isacoff ${ }^{*}, \dagger, \nabla, \bigcirc$ \\ † Department of Molecular and Cell Biology, University of California, Berkeley, California 94720, \\ United States \\ ‡ Department of Chemical Biology, Max Planck Institute for Medical Research, Jahnstraße 29, \\ 69120 Heidelberg, Germany \\ $\S$ Department of Biochemistry, Weill Cornell Medical College, New York, New York 10024, United \\ States \\ " Departments of Psychiatry \& Pharmacology, Columbia University, New York, New York 10032, \\ United States \\ ${ }^{\perp}$ Division of Molecular Therapeutics, New York State Psychiatric Institute, New York, New York \\ 10032, United States \\ \# Department of Chemistry, New York University, New York, New York 10003, United States \\ $\nabla$ Helen Wills Neuroscience Institute, University of California, Berkeley, California, 94720, United \\ States \\ ○ Molecular Biophysics \& Integrated Bioimaging Division, Lawrence Berkeley National Laboratory, \\ Berkeley, California 94720, United States
}

\begin{abstract}
G protein-coupled receptors (GPCRs) are membrane proteins that play important roles in biology. However, our understanding of their function in complex living systems is limited because we lack tools that can target individual receptors with sufficient precision. State-of-the-art approaches, including DREADDs, optoXRs, and PORTL gated-receptors, control GPCR signaling with molecular, cell type, and temporal specificity. Nonetheless, these tools are based on engineered non-native proteins that may (i) express at nonphysiological levels, (ii) localize and turnover incorrectly, and/or (iii) fail to interact with endogenous partners. Alternatively, membrane-
\end{abstract}

*Corresponding Author: ehud@berkeley.edu.

Author Contributions

P.C.D. and C.S. performed the molecular biology. J.B. performed the chemical synthesis. M.V. generated the AAV. P.C.D., V.V., and Z.F. performed the experiments. E.Y.I. supervised the project. P.C.D. and E.Y.I. wrote the manuscript with input from the other authors. All authors approved the final version of the manuscript.

The authors declare no competing financial interest.

ASSOCIATED CONTENT

The Supporting Information is available free of charge on the ACS Publications website at DOI: 10.1021/jacs.9b02895.

Supplemental figures and schemes, details of chemical synthesis, and methods (PDF) 
anchored ligands (t-toxins, DARTs) target endogenous receptors with molecular and cell type specificity but cannot be turned on and off. In this study, we used a combination of chemistry, biology, and light to control endogenous metabotropic glutamate receptor 2 (mGluR2), a Family C GPCR, in primary cortical neurons. mGluR2 was rapidly, reversibly, and selectively activated with photoswitchable glutamate tethered to a genetically targeted-plasma membrane anchor (membrane

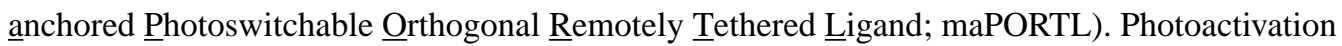
was tuned by adjusting the length of the PORTL as well as the expression level and geometry of the membrane anchor. Our findings provide a template for controlling endogenous GPCRs with cell type specificity and high spatiotemporal precision.

\section{Graphical Abstract}

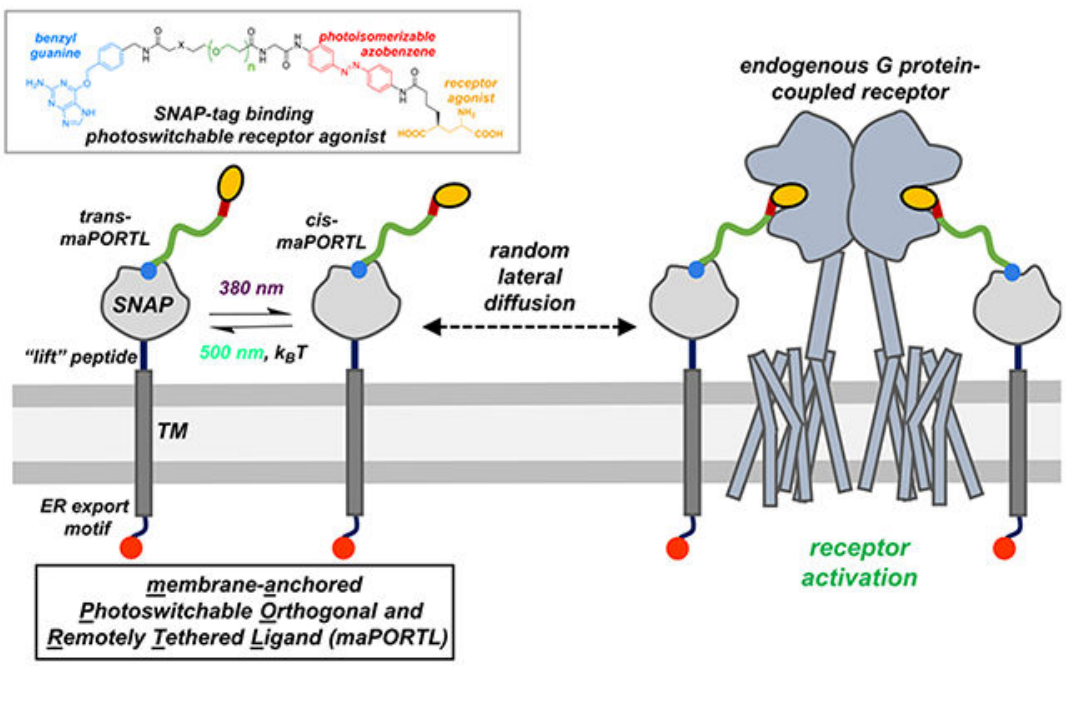

\section{INTRODUCTION}

G protein-coupled receptors (GPCRs) represent the largest superfamily of membrane proteins (>800 members). ${ }^{1}$ They respond to diverse stimuli (e.g., light, chemicals, peptides) and regulate a wide range of biological functions. ${ }^{1}$ Elucidating the roles of individual GPCRs is of profound importance for understanding physiological processes as well as pathological states in which GPCRs and/or their endogenous ligands are dysregulated. Moreover, GPCRs are targets of $\sim 25 \%$ of all currently available medications, ${ }^{2}$ and thus their characterization may shed light on mechanisms of drug action and enable the development of superior therapeutic strategies.

GPCRs are spatially organized and temporally activated in a complex manner in living systems (especially the brain), making it difficult to interrogate individual receptors with sufficient precision. Each GPCR can exist and have distinct roles in more than one location. Not only can a receptor be found in different organs and tissues, it can also be expressed in neighboring but distinct cell types within the same area. GPCRs can also be turned on and off by their endogenous ligands in hundreds of milliseconds to tens of seconds. ${ }^{3-5}$ Moreover, the precise temporal dynamics of GPCR activation can govern how a receptor controls downstream signaling processes and physiology. 6,7 
Traditional approaches that target GPCRs, including pharmacology (chemical antagonists, agonists, allosteric modulators) and genetics (knockouts, overexpression), face challenges of limited molecular, cell type, and/or spatiotemporal specificity. Thus, considerable effort has gone into the development of engineered GPCRs that can be turned on and off in genetically defined-cell types and locations. These approaches have employed chemogenetics (GPCRs designed to only respond to synthetic chemicals that do not exist in vivo; DREADDs ${ }^{8}$ ) or optogenetics (chimeric GPCRs consisting of a partial sequence of a receptor of interest and the transmembrane portion of the naturally light-sensitive components of rhodopsin; optoXRs $^{9-11}$ ).

DREADDs and optoXRs require substantial modification to the receptor sequence, which may limit their physiological relevance (e.g., in both cases the receptor is no longer able to bind its endogenous ligand). In contrast, we devised a photopharmacological approach that can be used to control full length, near-native GPCRs. ${ }^{12-15}$ We used the latest iteration of this approach, Photoswitchable Orthogonal Remotely Tethered Ligands (PORTLs), to rapidly and reversibly photoactivate metabotropic glutamate receptors (mGluRs), which are Family C GPCRs. ${ }^{14,15}$ We first synthesized a family of PORTLs that contain a receptor ligand (glutamate), an azobenzene moiety that rapidly photoisomerizes between its transand cis-isomers with specific wavelengths of light, as well as a benzylguanine (benzylguanine-azobenzene-glutamate; BGAG; Figures 1A and S1). The PORTL is covalently tethered via its benzylguanine to a genetically encoded SNAP-tag that we fused directly to the Nterminus of the receptor (SNAP-mGluR; Figure 1B). Several aspects of the PORTL approach are critical to its design. First, the glutamate moiety of BGAG was placed flush to the azobenzene (Figure 1A) so that it sterically binds the receptor in only one photoisomeric state (cis but not trans), thus allowing the PORTL to turn on and off the receptor in a lightdependent manner (Figure 1B). Second, tethering the PORTL affords selectivity by increasing its local concentration at the receptor while preventing its binding to off-target proteins. Third, because the PORTL is attached to SNAP-tag and is therefore remote from its binding site in the receptor, a long chemical linker was used to provide additional reach (Figure 1A and B). Finally, the SNAP-tagged receptor is genetically encoded and thus can be targeted to defined cell types.

PORTLs and the other engineered tools discussed above are a powerful means to control GPCR signaling in living systems. However, they all require modification to a GPCR's sequence that may alter receptor function and therefore provide an incomplete and possibly inaccurate view of its physiological role. Moreover, the delivery of these tools necessitates nonphysiological receptor overexpression or costly and timeconsuming genetic knock-in. Taking this into consideration, we sought to develop a strategy to control GPCRs with as much precision as that afforded by existing methods, but in a manner that does not modify the receptor, i.e., an approach that targets endogenous receptors.

Endogenous ion channels ${ }^{16}$ and more recently GPCRs ${ }^{17}$ have been targeted for selective pharmacological control using genetically encoded membrane-anchored peptidic ligands (ttoxins), which interact with their target protein as a result of a random encounter within the plasma membrane and high ligand affinity. ${ }^{18}$ This approach was recently adapted to block receptors that bind chemical ligands; that is, a HALO-tag was anchored to the plasma 
membrane and conjugated to a receptor antagonist (DARTs). ${ }^{19}$ Although t-toxins and DARTs can be targeted selectively to a desired cell type, once applied they act persistently until removed from the plasma membrane by the cell. To address this limitation, an ion channel t-toxin was recently engineered with a light-sensitive LOV domain (LumiToxin), ${ }^{20}$ enabling it to partially block channel activity in a reversible manner. Although the LumiToxin design could in principle be applied to peptide binding-GPCRs, it is not suitable for the large number of receptors that bind chemical ligands. ${ }^{21}$ Moreover, the reversal kinetics of LOV domains are slow ${ }^{20}$ compared to the millisecond to second time scales of fast and slow synaptic events.

In this study, we developed a system that combines the membrane anchor of a DART with the photoswitchable control of a PORTL to enable genetically targeted, reversible activation of a GPCR. We applied the approach to wildtype mGluR2 (mGluR2-WT), which plays important roles in brain circuits and is a putative target for the treatment of schizophrenia. ${ }^{22}$ Instead of tethering BGAG as a PORTL directly to an engineered version of the receptor (SNAP-mGluR; Figure 1B), we tethered it to a diffusible, membrane anchored SNAP-tag (membrane anchored Photoswitchable $\underline{\text { Orthogonal }}$ Remotely Tethered Ligand; maPORTL; Figure 1C). We optimized mGluR2-WT photoactivation with the maPORTL in HEK293T cells by adjusting (i) the chemical length of the BGAG linker, (ii) the positioning of the SNAP-tag relative to the plasma membrane, and (iii) the surface density membrane anchored SNAP-tag. The photoactivation of the unaltered, native receptor by BGAG tethered to a membrane anchor (the maPORTL) is as effective as that achieved by BGAG attached directly to the receptor. Furthermore, we demonstrate that the maPORTL selectively photoactivates endogenous mGluR2 in primary cortical neurons. Thus, the combination of genetic targeting of a membrane anchor and optical control of a tethered ligand gives the maPORTL approach the properties needed for the rapid, reversible, cell type specific, and spatially defined control of endogenous GPCRs in native tissue (ex vivo and in vivo). The maPORTL approach should be generally applicable to membrane proteins that are regulated by extracellular ligands.

\section{RESULTS AND DISCUSSION}

\section{Development of a Membrane Anchored SNAP-tag}

The maPORTL approach requires a membrane anchored SNAP-tag. To accomplish this, we fused SNAP-tag to a single-pass transmembrane segment (TM) to generate SNAP-TM. The TM was taken from low density lipoprotein receptor (LDLR), which supports structured extracellular elements with a single TM. ${ }^{23}$

For an maPORTL to control its target receptor, the photoswitchable ligand must reach from its anchor point in SNAP-TM to the receptor's ligand binding site (Figure 1C). mGluR2-WT is an obligatory dimer that binds glutamate in its extracellular ligand binding domain (LBD), resulting in conformational changes in the cysteine rich and transmembrane domains (CRD and TMD, respectively) that facilitate G protein binding and activation (Figure 1B). ${ }^{24,25}$ Thus, photoactivation of mGluR2-WT by SNAP-TM:BGAG is expected to require that (i) SNAP-TM is located in the same place that canonical receptor activation occurs (the plasma membrane), and (ii) the SNAP-tag of SNAP-TM is on the same face of the membrane as the 
LBD of mGluR2-WT (extracellular). To assess the cellular distribution of SNAP-TM, we labeled SNAP with the membrane permeant dye BG-TMR. We found that a large fraction of SNAP-TM resides on the plasma membrane (Figure S2A). We next labeled cells with the membrane impermeant dye BG-Alexa647 (Figure S2B), which confirmed that SNAP-tag is present on the extracellular face of the plasma membrane.

We hypothesized that because mGluR2-WT and SNAP-TM likely interact by random collision rather than through a specific interaction, SNAP-TM would need to be expressed in stoichiometric excess of mGluR2-WT in order for BGAG to be at a sufficient concentration to efficiently photoactivate the receptor. We estimated the relative ratio of mGluR2 and SNAP-TM by measuring BG-Alexa647 labeling of cells expressing SNAP-mGluR2 or SNAP-TM (Figure S2C). Our measurements indicated that surface expression of SNAP-TM is $\sim 3$-fold higher than the receptor (one-way ANOVA, Tukey, $p<0.0001$ ).

\section{mGluR2-WT Photoactivation by the maPORTL Depends on the Length of the Photoswitchable Ligand}

To measure agonist-induced activation of mGluR2, we used a $\mathrm{G}_{\mathrm{i} / \mathrm{o}}$-mediated $\mathrm{G}$ protein-gated inwardly rectifying potassium channel (GIRK) activation assay, ${ }^{12}$ whereby receptor activation evokes an inward current that is measured using whole-cell, voltage clamp recordings (Figure S3). We employed BGAGs containing either zero, 12, or 28 polyethylene glycol (PEG) repeats between the benzylguanine and azobenzene glutamate moieties $\left(\mathrm{BGAG}_{0}, \mathrm{BGAG}_{12}\right.$, or $\mathrm{BGAG}_{28}$; Figures $\mathrm{S} 1$ and $\left.2 \mathrm{~A}\right)$. The $\mathrm{BGAGs}$ switch from the trans to cis isomer configuration in response to illumination with near-UV light $(380 \mathrm{~nm})$ and vice versa with visible cyan light $(500 \mathrm{~nm}) .{ }^{14,15}$ Photoswitching either $\mathrm{BGAG}_{0}$ or $\mathrm{BGAG}_{12}$ tethered to SNAP-TM did not have a measurable effect on mGluR2-WT (compared to $1 \mathrm{mM}$ glutamate: $-1 \pm 1 \%, n=4$, and $1 \pm 1 \%, n=4$, respectively; Figure 2B and D). However, switching from the trans to cis in $\mathrm{BGAG}_{28}$ resulted in weak partial mGluR2-WT activation ( $13 \pm 1 \%$ of $1 \mathrm{mM}$ glutamate, $n=4$ ) that was reversed by switching back to the trans isomer (Figure $2 \mathrm{~B}$ and $\mathrm{D}$ ). We asked whether $\mathrm{BGAG}_{28}$ is more efficacious at mGluR2-WT because it binds SNAP-TM more efficiently than $\mathrm{BGAG}_{0}$ or $\mathrm{BGAG}_{12}$. However, there was no significant difference in the ability of the BGAGs to attach to SNAP-TM when compared to the binding of the fluorescent dye BG-Alexa647 (one-way ANOVA; Figure S4).

There was a striking contrast between the effect of linker length when BGAG was tethered to SNAP-TM and gated a separate mGluR2-WT protein versus when BGAG was tethered directly to SNAP-mGluR2. Consistent with our previous findings, when $\mathrm{BGAG}_{0}$ and $\mathrm{BGAG}_{12}$ were tethered directly to SNAP-mGluR2, they photoactivated the receptor to a similar degree ( $42 \pm 4 \%$ and $43 \pm 4 \%$ of $1 \mathrm{mM}$ glutamate, $n=8$ and 7, respectively, one-way ANOVA, Tukey, $p>0.5$; Figure $2 \mathrm{C}$ and $\mathrm{D}$ ). However, when $\mathrm{BGAG}_{28}$ was tethered directly to SNAP-mGluR2, it was significantly less effective ( $13 \pm 4 \%$ of $1 \mathrm{mM}$ glutamate, $n=8$, one-way ANOVA, Tukey, $p<0.0001$; Figure $2 \mathrm{C}$ and D). Thus, the BGAG with the longest linker was the weakest photoagonist of SNAP-mGluR2, yet it was the only compound that activated mGluR2-WT when tethered to SNAP-TM.

BGAG can activate SNAP-mGluR2 even with the shortest linker (zero PEGs; Figure 2), suggesting that the SNAP-to-LBD distance in this receptor is relatively short (Figure 1B). As 
BGAG gets longer, it explores a wider 3-dimensional space that results in a lower effective concentration of its glutamate moiety. ${ }^{11}$ Thus, the BGAG with the longest linker (28-PEGs) is associated with the weakest photoactivation of SNAP-mGluR2 (Figure 2). However, for BGAG to reach between two proteins (i.e., from an anchored SNAP-tag adjacent the plasma membrane to the glutamate binding site in the extracellular receptor LBD; Figure 1C), its linker must be longer: the only BGAG to work at all was that with the longest linker (Figure 2).

\section{mGluR2-WT Photoactivation Is Enhanced by Inserting a "Lift" Peptide into the Membrane Anchor}

We hypothesized mGluR2-WT photoactivation would be enhanced by bringing SNAP-tag and its bound BGAG closer to the LBD of mGluR2-WT. To accomplish this, we generated a series of SNAP-TM variants with "lift" peptides of varying lengths and physical properties inserted between the SNAP-tag and the TM. These included a flexible linker (GGGGS), a rigid $a$-helical linker (EAAAK), three repeats of the rigid linker (EAAAK) $)_{3},{ }^{26}$ and a much larger spacer element consisting of the fluorescent protein mVenus followed by an EAAAK linker (mVenus-EAAAK; Figure 3A).

Photoactivation with $\mathrm{BGAG}_{28}$ tethered to SNAP-EAAAK-TM (SNAP-EAAAKTM:BGAG 28 ) was significantly greater than with the SNAP-TM that lacked a lift peptide (one-way ANOVA, Tukey, $p<0.05$; Figure $3 \mathrm{C}$ and F), the greatest overall among the SNAP$\mathrm{TM}$ variants (Figure $3 \mathrm{~F}$ ), and not significantly different from $\mathrm{BGAG}_{0}$ attached directly to SNAP-mGluR2 (unpaired $t$ test, $\mathrm{p}=0.34$ ). Notably, photoactivation with SNAP-EAAAKTM:BGAG 28 was $\sim 2$-fold higher than that with GGGGS (one-way ANOVA, Tukey, $p<$ 0.05 ; Figure $3 \mathrm{~F}$ ), suggesting that the rigid linker is better than the flexible linker at stabilizing the SNAP:BGAG complex in a position that is appropriate for receptor activation.

Photoactivation by SNAP-TM variants with greater lift was either weak [(EAAAK $)_{3} ; 10 \pm$ $2 \%$ of $1 \mathrm{mM}$ glutamate, $n=3$; Figure 3D and F] or not observable (mVenus-EAAAK; $-1 \pm$ $2 \%$ of $1 \mathrm{mM}$ glutamate, $n=3$; Figure $3 \mathrm{E}$ and F), suggesting that BGAG must be optimally positioned above the plasma membrane. To test this further, we combined the longer SNAPTM variants, SNAP-(EAAAK) - $_{3}$ TM and SNAP-mVe-nus-EAAAK-TM, with the shorter $B G A G$ analogs $B A_{0}$ and $B_{12}$. In both cases, the most effective photoagonist was $\mathrm{BGAG}_{12}$, not $\mathrm{BGAG}_{28}$ (Figure S5).

BGAG must be a minimal length to be able to reach from its anchor point in SNAP-TM at the plasma membrane to its extracellular binding site in the mGluR2-WT LBD (Figure 2). Extending the maPORTL by inserting a lift peptide into SNAP-TM brings BGAG closer to the LBD, increasing its effective concentration (Figure 3). However, further increasing the length of the maPORTL is eventually counterproductive (Figure S5) because the larger 3dimensional space explored by the ligand results in a lower effective concentration.

\section{mGluR2-WT Photoactivation with the maPORTL Is Rapid, Reversible, and Repeatable}

Consistent with the photophysical properties of typical azobenzenes, ${ }^{27}$ photoactivation with SNAP-EAAAK-TM:BGAG 28 was bistable (Figure 4A); that is, a short flash of near-UV light triggered persistent activation, and a short flash of cyan light triggered persistent 
deactivation. The kinetics of activation and deactivation were rapid (Figure 4B). Moreover, photoactivation could be reversed and repeated with no significant loss in magnitude after multiple cycles ( $n=3$, RM one-way ANOVA, $p=0.64$; Figure $4 \mathrm{C}$ and D). Thus, like DARTs, maPORTLs can be used to persistently ligand a target receptor, mimicking the actions of drugs as well as pathological states. In addition, unlike DARTs, maPORTLs can also be reversibly turned on and off, a feature that can be used to mimic the actions of endogenous ligands such as glutamate that rapidly rise and fall in concentration in living systems.

\section{mGluR2 Photoactivation Is Limited by the Density of the maPORTL}

We improved mGluR2-WT photoactivation by adjusting the positioning of the maPORTL at the cell surface (Figures 2 and 3). However, even the most effective maPORTL, SNAPEAAAK-TM: $\mathrm{BGAG}_{28}$, only partially activated the receptor (Figure 3 ). One possibility is that the maPORTL is a partial agonist relative to glutamate. Alternatively, the maPORTL could be a full agonist that is at too low of a density at the plasma membrane to fully occupy the receptor. The activation of an mGluR2 dimer is a cooperative process, whereby the binding of one glutamate activates very partially and two glutamates more than double the activation. ${ }^{28-30}$ Thus, full receptor activation is expected to require that the dimer simultaneously binds two SNAP-TM:BGAG complexes.

To determine whether mGluR2-WT photoactivation is limited by partial agonism or partial occupancy, we photoactivated the receptor with SNAP-EAAAK-TM:BGAG 28 in the presence of an $\sim$ EC50 concentration of glutamate $(1 \mu \mathrm{M}$; Figures S6A and B). A partial photoagonist that fully occupies the receptor is expected to compete with and possibly inhibit $1 \mu \mathrm{M}$ glutamate-induced mGluR2-WT activation, whereas a full photoagonist that partially occupies the receptor should further activate it. SNAP-EAAAK-TM:BGAG 28 was able to photoactivate mGluR2-WT even in the presence of $1 \mu \mathrm{M}$ glutamate (Figure S6C), indicating that photoactivation is limited because some receptors are not occupied or are only partially occupied by ligand.

\section{mGluR2-WT Photoactivation Is Enhanced by Increasing the Density of the maPORTL at the Plasma Membrane}

We sought to enhance photoactivation with the maPORTL approach by increasing the availability of BGAG, analogous to when we doubled the number of azobenzene-glutamates directly tethered to mGluR2. ${ }^{15}$ To accomplish this, we generated a SNAP-TM variant with two SNAP-tags in tandem (tSNAP-EAAAK-TM) (Figure 5A) so that two BGAGs attached to a single anchor could simultaneously bind — and thus fully activate-the receptor. We also sought to increase the density of SNAP-TM at the cell surface by increasing its export from intracellular membranes (Figure S2A) to the plasma membrane. We added to the C-terminus of SNAP-EAAAK-TM and ISNAP-EAAAK-TM an endoplasmic reticulum export motif that was taken from the potassium channel Kir2.1 (ISNAP-EAAAK-TM-ERE; Figure 5A). This motif has been shown to enhance surface expression when transplanted into other membrane proteins, including halorhodopsin. ${ }^{31}$ 
We first tested whether these modifications resulted in a greater number of SNAP-tags on the plasma membrane. Cells expressing variants of SNAP-TM were labeled with the membrane impermeant fluorescent dye BG-Alexa647. Compared to SNAP-TM and SNAPEAAAK-TM, fluorescence was higher with $t$ SNAP-EAAAK-TM ( 2-fold), SNAPEAAAK-TM-ERE ( $\sim$-fold), and $t$ SNAP-EAAAK-TM-ERE ( $\sim$-fold; $n=10$, one-way ANOVA, Tukey, $p<0.05$; Figure 5C), indicating an increase in the number of SNAP-tags at the cell surface and thus the available binding sites for BGAG. There was no significant difference in cell viability between the SNAP-TM variants ( $n=3$, one-way ANOVA, Tukey, $p>0.05$; Figure S7). Furthermore, survival was greater than 95\% (Figure S7), indicating that the membrane anchor is well tolerated in cells.

Photoactivation of mGluR2-WT progressively increased with increasing levels of surface SNAP-tag (Figure 5D-H). The greatest photoactivation of mGluR2-WT overall was observed with $t$ SNAP-EAAAK-TM-ERE:BGAG 28 (69 $\pm 8 \%$ of 1 mM glutamate, $n=6$; Figures $5 \mathrm{G}$ and $\mathrm{H}$ ), which was $\sim 2$-fold greater than that with SNAP-EAAAK-TM:BGAG 28 (one-way ANOVA, Tukey, $p<0.01$; Figures $5 \mathrm{G}$ and $\mathrm{H}$ ). Notably, photoactivation with ISNAP-EAAAK-TM-ERE:BGAG 28 also exceeded what was achieved with $\mathrm{BGAG}_{0}$ attached directly to SNAP-mGluR2 ( 2-fold; one-way ANOVA, Tukey, $p<0.05$; Figure 5G). Overall, these results indicate that increasing the number of SNAP-tags fused to the TM as well as the surface expression of SNAP-TM enhances photoactivation of mGluR2-WT.

\section{maPORTL Photoactivation Is Selective for mGluR Subtype}

PORTLs are selective because they are physically restricted to their target receptor (Figure 1B). In contrast, maPORTLs are not constrained to any given membrane protein. Thus, the degree of receptor selectivity of the maPORTL approach is expected to depend on the binding properties of the photoswitchable ligand and its positioning relative to the receptor binding site.

Because the SNAP-TM:BGAG maPORTL contains glutamate, it could also act on other mGluRs, some of which may be coexpressed with mGluR2 in the same cell. For example, dorsal root ganglion neurons coexpress mGluR2 and mGluR3. ${ }^{32}$ However, we showed previously that as a PORTL, $\mathrm{BGAG}_{28}$ activates SNAP-mGluR2 but has little or no effect on its closest relative, the other Group II member mGluR3, or on Group III members mGluR4, 7, and $8 .{ }^{15}$ Consistent with these findings, $t$ SNAP-EAAAK-TM-ERE:BGAG 28 had no effect on mGluR3-WT, mGluR4-WT, or mGluR8-WT (Figures S8). Furthermore, it very weakly photoactivated mGluR7-WT as a trans-agonist $(8 \pm 4 \%$ of $1 \mathrm{mM}$ glutamate, $\mathrm{n}=3$; Figures S8C and F), analogous to the effects of BGAG at SNAP-mGluR7. ${ }^{15}$ iSNAP-EAAAK-TMERE:BGAG 28 also had no effect on mGluR1-WT, a Group I mGluR (Figure S8). tSNAPEAAAK-TM-ERE expression was not significantly different in cells with mGluR2-WT and any other mGluR subtype (one-way ANOVA, Tukey; Figure S8G), indicating that the selective activation of mGluR2-WT by the maPORTL is not due to differences in surface SNAP-tag levels.

tSNAP-EAAAK-TM-ERE:BGAG 28 could also have effects on ionotropic glutamate receptors (iGluRs). However, this would appear to be unlikely given that BGAG contains a 4-methyl-substituted glutamate, which has low affinity for iGluRs. ${ }^{33,34}$ To evaluate this, we 
tested ISNAP-EAAAK-TM-ERE:BGAG ${ }_{28}$ on selected variants from each major class of iGluR [AMPAR (GluRA1), NMDAR (GluN1/GluN2B), and KAR (GluK2)] and found there to be no effect on any of these receptors (Figure S9). Although the maPORTL was not tested at every glutamate binding-protein, our results indicate that it preferentially photoactivates mGluR2-WT.

\section{Selective Photoactivation of Endogenous mGluR2 in Primary Cortical Neurons with a maPORTL}

Having shown that the maPORTL approach works in HEK293T cells in which mGluR2 is overexpressed, we set out to determine if it would work in neurons that express mGluR2 naturally. We turned to cultured rat primary cortical neurons (CNs), which express mGluR2 endogenously. ${ }^{35}$ Antibody staining revealed that mGluR2 is distributed in a punctate manner throughout the CNs (Figure 6A), consistent with its expression pattern in ex vivo preparations of rat cortex. ${ }^{36}$ To deliver SNAP-EAAAK-TM-ERE to CNs, we generated an adeno-associated virus (AAV) encoding this construct under the CAG promoter followed by the self-cleavable peptide P2A and the fluorescent reporter protein mVenus (Figure 6B). AAV-infected neurons were labeled with BG-Alexa647, which indicated robust surface expression of SNAP-EAAAK-TM-ERE throughout the cell soma and neurites (Figure 6B).

To determine whether endogenous mGluR2 can be photoactivated, we measured the spontaneous activity of $\mathrm{BGAG}_{28}$-labeled, mVenus-expressing CNs using whole-cell, current-clamp recordings. In response to near-UV light, there was robust suppression of spontaneous firing that was reversible (Figure 6C and D), repeatable (Figure 6C and D), and bistable (Figure 6E). Photoactivation resulted in a $4 \pm 1 \mathrm{mV}(n=8)$ hyperpolarization (Figure $6 \mathrm{E}$ ), consistent with the effects of endogenous $\mathrm{G}_{\mathrm{i} / \mathrm{o}}$-coupled receptor activation in cortical neurons. ${ }^{37}$ This effect was not observed in uninfected $\mathrm{CNs}$ labeled with $\mathrm{BGAG}_{28}$ (Figures S10A and B). Moreover, there was no effect of $\mathrm{BGAG}_{28}$ on uninfected CNs even if the compound was left in solution (Figures $\mathrm{S} 10 \mathrm{C}$ and D), indicating that $1 \mu \mathrm{M} \mathrm{BGAG}_{28}$ in solution has too low an encounter rate with receptor to produce detectable activation.

The effect of SNAP-EAAAK-TM-ERE:BGAG 28 on CNs was comparable to that by the mGluR2/3 agonist LY379268 (Figures S10E-G). ${ }^{35}$ Furthermore, there was no photoeffect in the presence of the mGluR2/3 antagonist LY341495 (Figures S10H and I). ${ }^{35}$ These results indicate that Group II mGluRs are the target of SNAP-EAAAK-TM-ERE:BGAG 28 in CNs.

Because CNs coexpress mGluR2 and mGluR3, ${ }^{35}$ it is possible that the observed photoeffect is mediated by one or both of these receptors. We saw no effect of the maPORTL on mGluR3 in HEK293T cells (Figure S8), but this may not be the case if the subcellular localization of receptor and SNAP-TM is different in neurons. To evaluate this, we used the selective mGluR3 negative allosteric modulator (NAM) ML337, which completely abolishes mGluR3 activation (Figures S11A and B). ${ }^{38}$ ML337 had no effect on SNAP-EAAAK-TMERE: $\mathrm{BGAG}_{28}$-induced inhibition of CNs (Figures S11C-E), supporting the interpretation that the target of the maPORTL in CNs is indeed mGluR2. 


\section{Effects of maPORTLs on Baseline mGluR2-WT Activation}

Increasing the level of BGAG at the cell surface enhances maximal mGluR2-WT

photoactivation because there is a higher availability of the cis-isomer under near-UV light.

However, because a small fraction of azobenzene is in cis instead of trans when visible light is used to rapidly turn the photoswitch off, ${ }^{27}$ a high BGAG concentration could, in principle, increase baseline receptor activation until the azobenzene fully relaxes into the trans state, as it does over tens of minutes in the dark. ${ }^{39}$ This could be problematic in contexts where it is difficult to control the amount of maPORTL in a target cell type in vivo.

To understand this effect, we modeled maPORTL-induced mGluR2-WT activation under visible light and near-UV light based on (i) the expression and functional effects associated with our existing SNAP-TM variants in HEK293T cells (Figure 5H) and (ii) the assumption that the amount of $\mathrm{BGAG}_{28}$ in the cis-state is 10 -fold greater under near-UV light than under visible light. ${ }^{27}$ This analysis suggested that baseline receptor activation under visible light is negligible for all SNAP-TM variants (Figures S12A and B). This included tSNAP-EAAAKTM-ERE, which is associated with the highest surface SNAP-tag levels observed in HEK293T cells ( $\sim 5$-fold greater than SNAP-TM; Figures 5H and S12B).

We tested this prediction by using the mGluR2 antagonist LY341495, which blocks receptor photoactivation. ${ }^{14}$ LY341495 had no effect on HEK293T cells expressing mGluR2-WT alone, consistent with its actions as a neutral antagonist (Figures S12C and D). ${ }^{40}$ As expected, LY341495 completely blocked the activation of mGluR2-WT by tSNAP-EAAAKTM-ERE:BGAG 28 under near-UV light (Figure S12E). However, it had no effect under visible light (Figures S12E and F), indicating that baseline receptor activity is not elevated in HEK293T cells. Similarly, under visible light, LY341495 had no effect on the spontaneous firing of CNs expressing SNAP-EAAAK-TM-ERE:BGAG 28 (Figures $\mathrm{S} 12 \mathrm{G}$ and $\mathrm{H}$ ).

The maPORTL did not increase baseline receptor activity in two different cellular backgrounds, even when the membrane anchor was driven by a strong expression promoter (e.g., see Figure 6B). Still, since extremely high maPORTL densities would result in baseline receptor activation (Figure S12A), we evaluated whether we could use as an maPORTL BGAG $12,460,{ }^{14}$ which contains a red-shifted azobenzene that transitions from its trans- to cis-isomer under blue light and relaxes back to trans in the dark much more rapidly (hundreds of milliseconds). ${ }^{14,41,42}$ We first evaluated $t$ SNAP-EAAAK-TM-

ERE:BGAG 12,460 and found there to be no effect on mGluR2-WT (Figures S13A and C), possibly because the ligand is not long enough to reach the receptor LBD. However, BGAG $_{12,460}$ photoactivated mGluR2-WT when tethered to a version of SNAP-TM with a longer lift peptide, SNAP-(EAAAK) 3 -TM (Figures S13B and C). Thus, a rapidly relaxing

photoswitchable ligand could be used to avoid prolonged baseline receptor activation under conditions of very high maPORTL density.

\section{CONCLUSION}

Obtaining a clear view of GPCR function is of profound importance for understanding physiology and disease. A major challenge is that many GPCRs have distinct roles in multiple tissue regions and cell types. Moreover, their temporal profiles of activation are 
complex, especially in the nervous system. Therefore, indiscriminately targeting GPCRs would obscure their function. To address these issues, substantial effort has gone toward engineering methods to control receptor activity in defined cells, locations, and times. However, these approaches (i) require modifications that likely alter receptor function (DREADDs, optoXRs, and PORTL-gated receptors), (ii) control endogenous receptors chronically in a manner that cannot be regulated (DARTs), or (iii) are limited to controlling endogenous peptide binding-receptors with slow reversal kinetics (LumiToxins). The consequence is that, to date, there has been no method for rapidly and reversibly controlling endogenous GPCRs with cell type specificity.

Here we devised a system that allows for selective, cell type specific, and spatiotemporally precise control of an endogenous mGluR by tethering a photoswitchable ligand to a membrane-anchored SNAP-tag, the maPORTL system. Optical control of mGluR2 by maPORTLs was optimized by tailoring the length of the linker between the SNAP attachment site and the receptor ligand, the spacer between the SNAP-tag and TM in the maPORTL's membrane anchor, the number of SNAP-tags on the membrane anchor, and the efficiency of delivery of the membrane anchor to the cell surface. We expect additional optimization will be necessary for receptors with differing structural architectures. For example, whereas Family C GPCRs, such as mGluRs, bind ligands in a large extracellular clamshell LBD, Family A GPCRs bind ligands in their TMD. Thus, we predict that shorter chemical linkers and/or peptide linkers in SNAP-TM will be required to maximize optical control of these receptors.

We used the maPORTL approach to target mGluR2, a GPCR that naturally binds chemical ligands. However, maPORTLs can likely be expanded to other types of GPCRs, considering that azobenzene has been conjugated to structurally and functionally diverse molecules such as peptides ${ }^{43}$ and fatty acids. ${ }^{44,45}$ maPORTLs are also expected to be compatible with synthetic ligands that bind orphan GPCRs as well as with allosteric modulators and biased ligands. Overall, we expect the maPORTL approach to be generalizable to a wide variety of physiologically and clinically important membrane proteins that bind extracellular ligands, including GPCRs, ion channels, and receptor-linked enzymes.

\section{Supplementary Material}

Refer to Web version on PubMed Central for supplementary material.

\section{ACKNOWLEDGMENTS}

This work was supported by NIH grants DA044696 to P.C.D., 2PN2EY018241 to E.Y.I. and D.T., R01 MH54137 to J.A.J., an R35 grant from the National Institute of General Medical Sciences (1 R35 GM124731) to J.L., as well as the ERC [Advanced Grant to D.T. (268795)].

\section{REFERENCES}

(1). Katritch V; Cherezov V; Stevens RC Annu. Rev. Pharmacol. Toxicol 2013, 53, 531. [PubMed: 23140243]

(2). Overington JP; Al-Lazikani B; Hopkins AL Nat. Rev. Drug Discovery 2006, 5, 993. [PubMed: 17139284] 
(3). Sun F; Zeng J; Jing M; Zhou J; Feng J; Owen SF; Luo Y; Li F; Wang H; Yamaguchi T; Yong Z; Gao Y; Peng W; Wang L; Zhang S; Du J; Lin D; Xu M; Kreitzer AC; Cui G; Li Y Cell 2018, 174, 481. [PubMed: 30007419]

(4). Patriarchi T; Cho JR; Merten K; Howe MW; Marley A; Xiong WH; Folk RW; Broussard GJ; Liang R; Jang MJ; Zhong H; Dombeck D; von Zastrow M; Nimmerjahn A; Gradinaru V; Williams JT; Tian L Science 2018, 360, eaat4422 [PubMed: 29853555]

(5). Adams PR; Brown DA J. Physiol 1982, 332, 263. [PubMed: 6984074]

(6). Sumit M; Neubig RR; Takayama S; Linderman JJ Integrative biology: quantitative biosciences from nano to macro 2015, 7, 1378. [PubMed: 26374065]

(7). Grundmann M; Kostenis E Trends Pharmacol. Sci 2017, 38, 1110. [PubMed: 29074251]

(8). Urban DJ; Roth BL Annu. Rev. Pharmacol. Toxicol 2015, 55, 399. [PubMed: 25292433]

(9). Airan RD; Thompson KR; Fenno LE; Bernstein H; Deisseroth K Nature 2009, 458, 1025. [PubMed: 19295515]

(10). Kim JM; Hwa J; Garriga P; Reeves PJ; RajBhandary UL; Khorana HG Biochemistry 2005, 44, 2284. [PubMed: 15709741]

(11). Siuda ER; McCall JG; Al-Hasani R; Shin G; Il Park S; Schmidt MJ; Anderson SL; Planer WJ; Rogers JA; Bruchas MR Nat. Commun 2015, 6, 8480. [PubMed: 26412387]

(12). Levitz J; Pantoja C; Gaub B; Janovjak H; Reiner A; Hoagland A; Schoppik D; Kane B; Stawski P; Schier AF; Trauner D; Isacoff EY Nat. Neurosci 2013, 16, 507. [PubMed: 23455609]

(13). Donthamsetti PC; Winter N; Schonberger M; Levitz J; Stanley C; Javitch JA; Isacoff EY; Trauner DJ Am. Chem. Soc 2017, 139, 18522.

(14). Broichhagen J; Levitz J; Sokol K; Leippe P; Konrad D; Isacoff EY; Trauner D; Damijonaitis A ACS Central Science 2015, 1, 383. [PubMed: 27162996]

(15). Levitz J; Broichhagen J; Leippe P; Konrad D; Trauner D; Isacoff EY Proc. Natl. Acad. Sci. U. S. A 2017, 114, No. E3546.

(16). Miwa JM; Ibanez-Tallon I; Crabtree GW; Sanchez R; Sali A; Role LW; Heintz N Neuron 1999, 23, 105. [PubMed: 10402197]

(17). Fortin JP; Zhu Y; Choi C; Beinborn M; Nitabach MN; Kopin AS Proc. Natl. Acad. Sci. U. S. A 2009, 106, 8049. [PubMed: 19416829]

(18). Ibanez-Tallon I; Nitabach MN Curr. Opin. Neurobiol 2012, 22, 72. [PubMed: 22119144]

(19). Shields BC; Kahuno E; Kim C; Apostolides PF; Brown J; Lindo S; Mensh BD; Dudman JT; Lavis LD; Tadross MR Science 2017, 356, eaaj2161 [PubMed: 28385956]

(20). Schmidt D; Tillberg PW; Chen F; Boyden ES Nat. Commun 2014, 5, 3019. [PubMed: 24407101]

(21). Gloriam DE; Fredriksson R; Schioth HB BMC Genomics 2007, 8, 338. [PubMed: 17892602]

(22). Weinberger DR Nat. Med 2007, 13, 1018. [PubMed: 17828217]

(23). Jeon H; Blacklow SC Annu. Rev. Biochem 2005, 74, 535. [PubMed: 15952897]

(24). Conn PJ; Pin JP Annu. Rev. Pharmacol. Toxicol 1997, 37, 205. [PubMed: 9131252]

(25). Niswender CM; Conn PJ Annu. Rev. Pharmacol. Toxicol 2010, 50, 295. [PubMed: 20055706]

(26). Chen X; Zaro JL; Shen WC Adv. Drug Delivery Rev 2013, 65, 1357.

(27). Beharry AA; Woolley GA Chem. Soc. Rev 2011, 40, 4422. [PubMed: 21483974]

(28). Kniazeff J; Bessis AS; Maurel D; Ansanay H; Prezeau L; Pin JP Nat. Struct. Mol. Biol 2004, 11, 706. [PubMed: 15235591]

(29). Vafabakhsh R; Levitz J; Isacoff EY Nature 2015, 524, 497. [PubMed: 26258295]

(30). Levitz J; Habrian C; Bharill S; Fu Z; Vafabakhsh R; Isacoff EY Neuron 2016, 92, 143. [PubMed: 27641494]

(31). Gradinaru V; Thompson KR; Deisseroth K Brain Cell Biol. 2008, 36, 129. [PubMed: 18677566]

(32). Sheahan TD; Valtcheva MV; McIlvried LA; Pullen MY; Baranger DAA; Gereau R. W. t. eNeuro 2018, 5, ENEURO.0412-17.2018

(33). Hansen JJ; Nielsen B; Krogsgaard-Larsen P; Brehm L; Nielsen EO; Curtis DR J. Med. Chem 1989, 32, 2254. [PubMed: 2552114]

(34). Robinson MB; Crooks SL; Johnson RL; Koerner JF Biochemistry 1985, 24, 2401. [PubMed: 2990528] 
(35). Koga K; Iwahori Y; Ozaki S; Ohta HJ Neurosci. Res 2010, 88, 2252.

(36). Bragina L; Bonifacino T; Bassi S; Milanese M; Bonanno G; Conti F Front Cell Neurosci 2015, 9 , 345. [PubMed: 26388733]

(37). van Aerde KI; Qi G; Feldmeyer D Cereb Cortex 2015, 25, 772. [PubMed: 24108800]

(38). Wenthur CJ; Morrison R; Felts AS; Smith KA; Engers JL; Byers FW; Daniels JS; Emmitte KA; Conn PJ; Lindsley CW J. Med. Chem 2013, 56, 5208. [PubMed: 23718281]

(39). Gorostiza P; Volgraf M; Numano R; Szobota S; Trauner D; Isacoff EY Proc. Natl. Acad. Sci. U. S. A 2007, 104, 10865. [PubMed: 17578923]

(40). Kingston AE; Ornstein PL; Wright RA; Johnson BG; Mayne NG; Burnett JP; Belagaje R; Wu S; Schoepp DD Neuropharmacology 1998, 37, 1. [PubMed: 9680254]

(41). Kienzler MA; Reiner A; Trautman E; Yoo S; Trauner D; Isacoff EY J. Am. Chem. Soc 2013, 135, 17683. [PubMed: 24171511]

(42). Carroll EC; Berlin S; Levitz J; Kienzler MA; Yuan Z; Madsen D; Larsen DS; Isacoff EY Proc. Natl. Acad. Sci. U. S. A 2015, 112, No. E776.

(43). Behrendt R; Schenk M; Musiol HJ; Moroder LJ Pept. Sci 1999, 5, 519.

(44). Frank JA; Moroni M; Moshourab R; Sumser M; Lewin GR; Trauner D Nat. Commun 2015, 6, 7118. [PubMed: 25997690]

(45). Frank JA; Franquelim HG; Schwille P; Trauner DJ Am. Chem. Soc 2016, 138, 12981. 

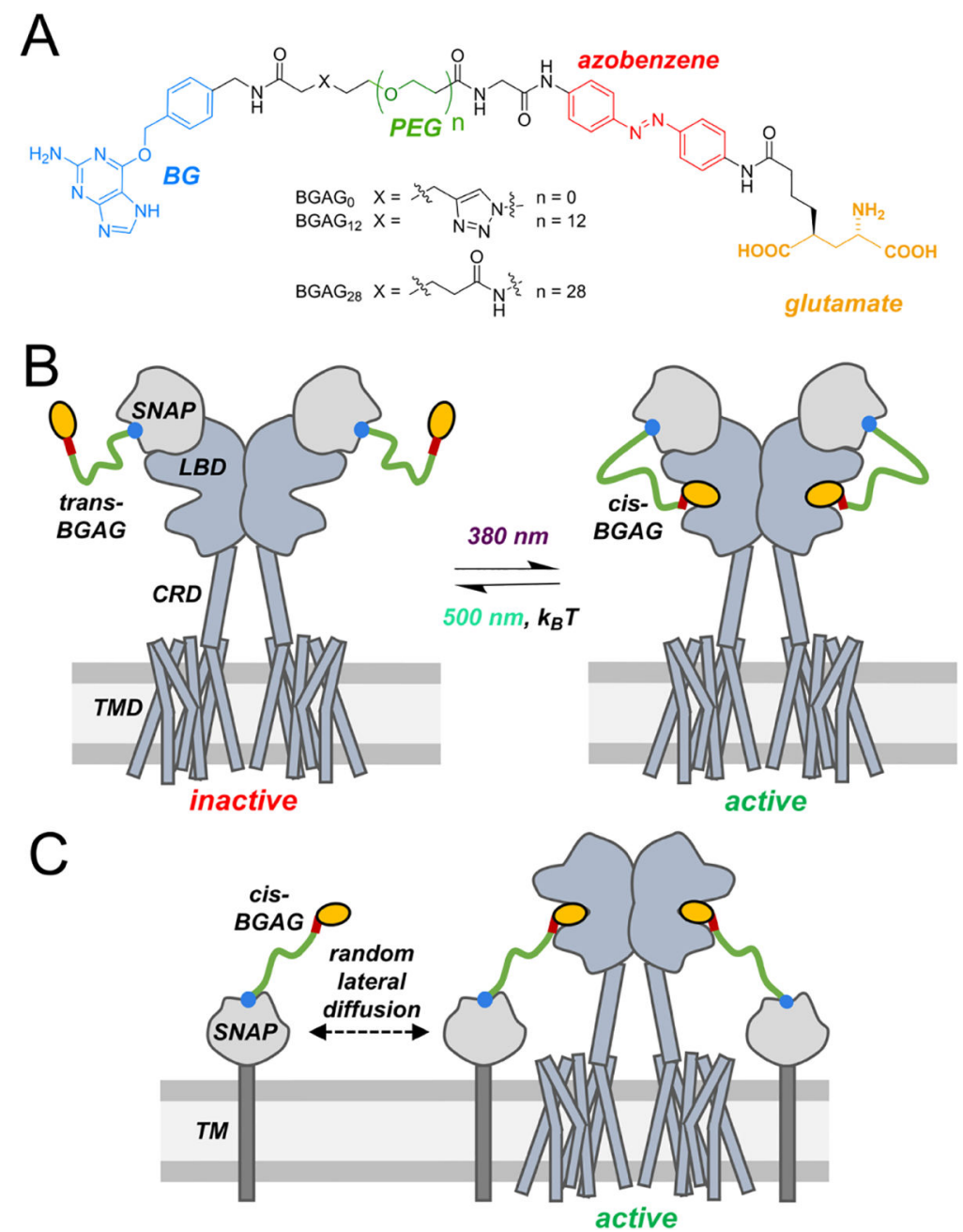

Figure 1.

Targeting modified and unmodified, native mGluRs with BGAG. (A) Benzylguanineazobenzene-glutamate (BGAG) with a polyethylene glycol (PEG) linker of varying lengths between benzylguanine (BG) and azobenzene-glutamate. " $\mathrm{X}$ " denotes distinct spacer elements within different analogs of BGAG. Refer to Figure $\mathrm{S} 1$ for full chemical structures. (B) mGluRs consist of a ligand binding domain (LBD), cysteine rich domain (CRD), and transmembrane domain (TMD). Photoisomerizable BGAG reversibly activates an mGluR with light when bound to a SNAP-tag fused to the receptor's LBD. (C) The active isomer of BGAG, cis-BGAG, tethered to a SNAP-tag fused to a single-pass transmembrane segment (SNAP-TM) randomly collides with and photoactivates a native (unmodified) mGluR. 

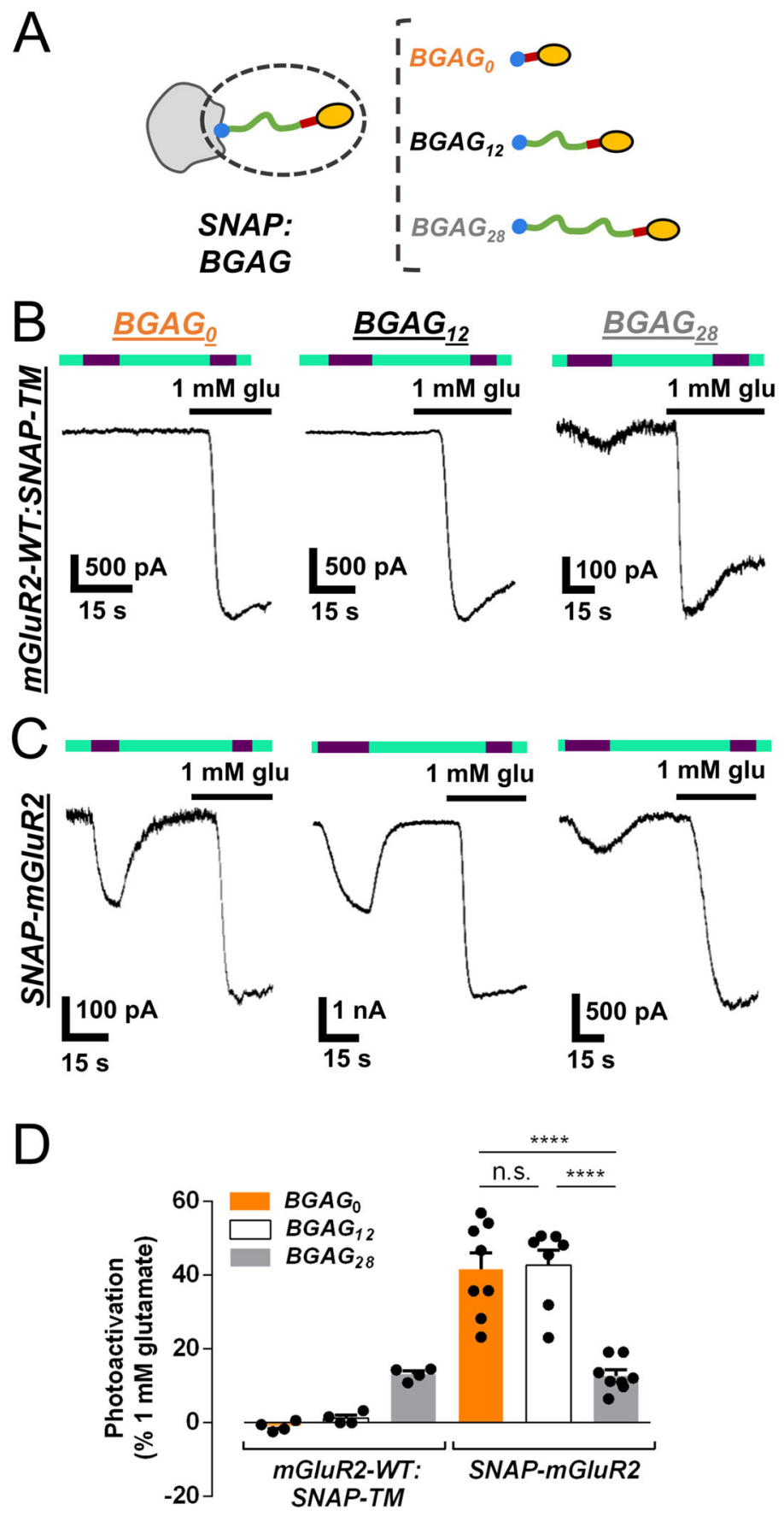

Figure 2.

Photoactivation depends on the length of the chemical linker in BGAG. (A) Schematic representation of SNAP-tag labeled with BGAG analogs with either zero, 12, or 28 PEG repeats. (B) Switching from 500 to $380 \mathrm{~nm}$ light (cyan and purple bars, respectively) resulted in photoactivation of $\mathrm{mGluR}^{2}-\mathrm{WT}$ with $\mathrm{BGAG}_{28}$ (right) but not $\mathrm{BGAG}_{0}$ (left) or BGAG $_{12}$ (center) tethered to SNAP-TM. (C) SNAP-mGluR2 was photoactivated to a lesser degree with $\mathrm{BGAG}_{28}$ (right) than $\mathrm{BGAG}_{0}$ (left) or $\mathrm{BGAG}_{12}$ (center). (D) Summary of photoactivation of mGluR2-WT coexpressed with SNAP-TM, or SNAP-mGluR2, by 
BGAGs of increasing length. Cells were labeled with BGAG analogs for $1 \mathrm{~h}$ at $37^{\circ} \mathrm{C}$. Oneway ANOVA, Tukey, $* * * * p<0.0001$. 

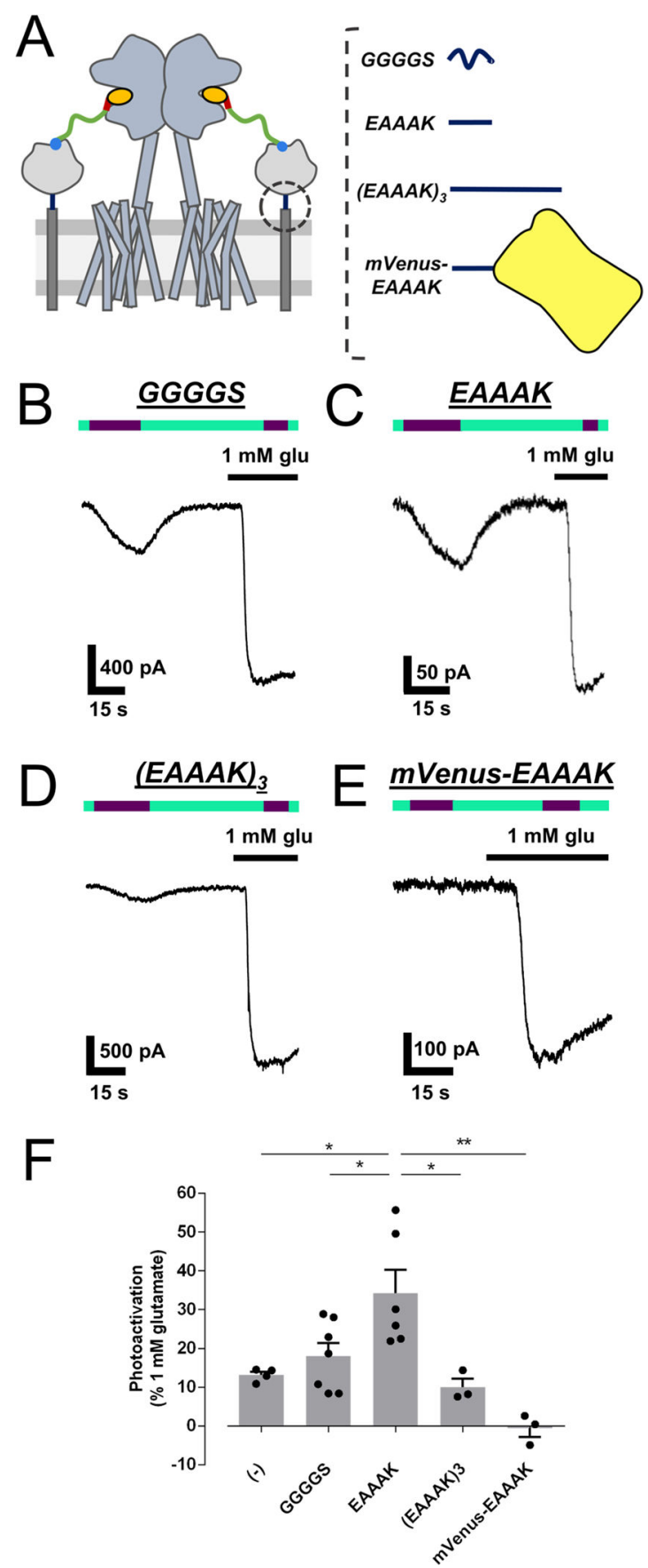

Figure 3.

Photoactivation depends on the properties of the peptide linker in SNAP-TM. (A) SNAP-TM variants with different "lift" peptides. (B-E) Representative trace of $\mathrm{BGAG}_{28}$-induced photoactivation of mGluR2-WT when tethered to (B) SNAP-GGGGS-TM, (C) SNAPEAAAK-TM, (D) SNAP-(EAAAK) 3 -TM, or (E) SNAP-mVenus-TM. (F) Summary of photoactivation of mGluR2-WT with SNAP-TM variants tethered to $\mathrm{BGAG}_{28}$. one-way ANOVA, Tukey, ${ }^{*} p<0.05, * * p<0.01$. 

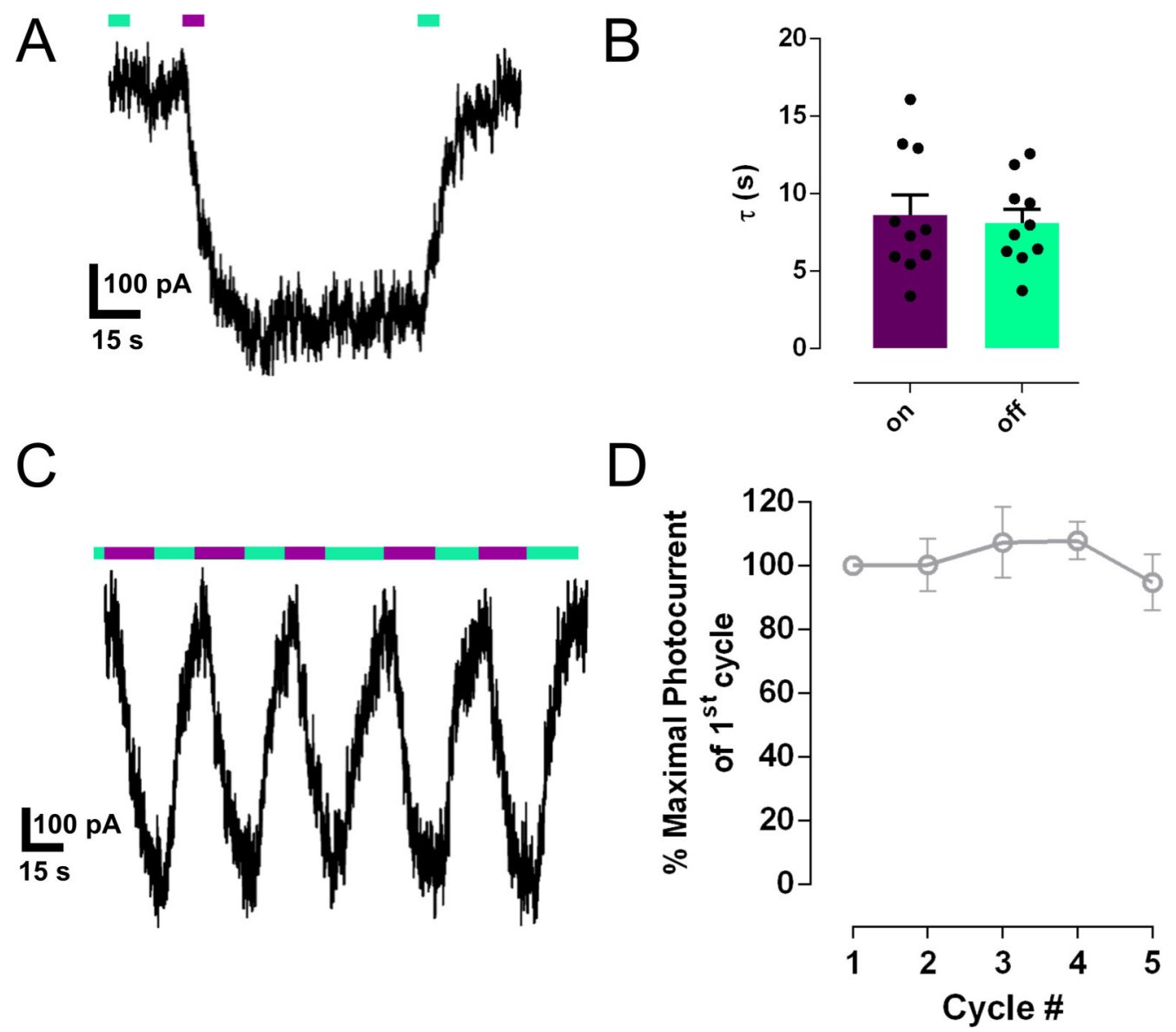

Figure 4.

Functional characterization of $\mathrm{BGAG}_{28}$-induced photoactivation of mGluR2-WT when tethered to SNAP-EAAAK-TM. (A) Short flashes (5 s) of near-UV and cyan light (380 and $500 \mathrm{~nm}$ ) were sufficient to stably activate and deactivate mGluR2-WT with $B^{-} G_{28}$, consistent with the bistability of azobenezene-containing ligands. (B) Kinetics of mGluR2WT photoactivation and deactivation. (C and D) mGluR2-WT was reversibly and repeatedly photoactivated over five cycles with no significant loss in maximal photoactivation across cycles $(n=3$, RM one-way ANOVA, $p=0.64)$. 

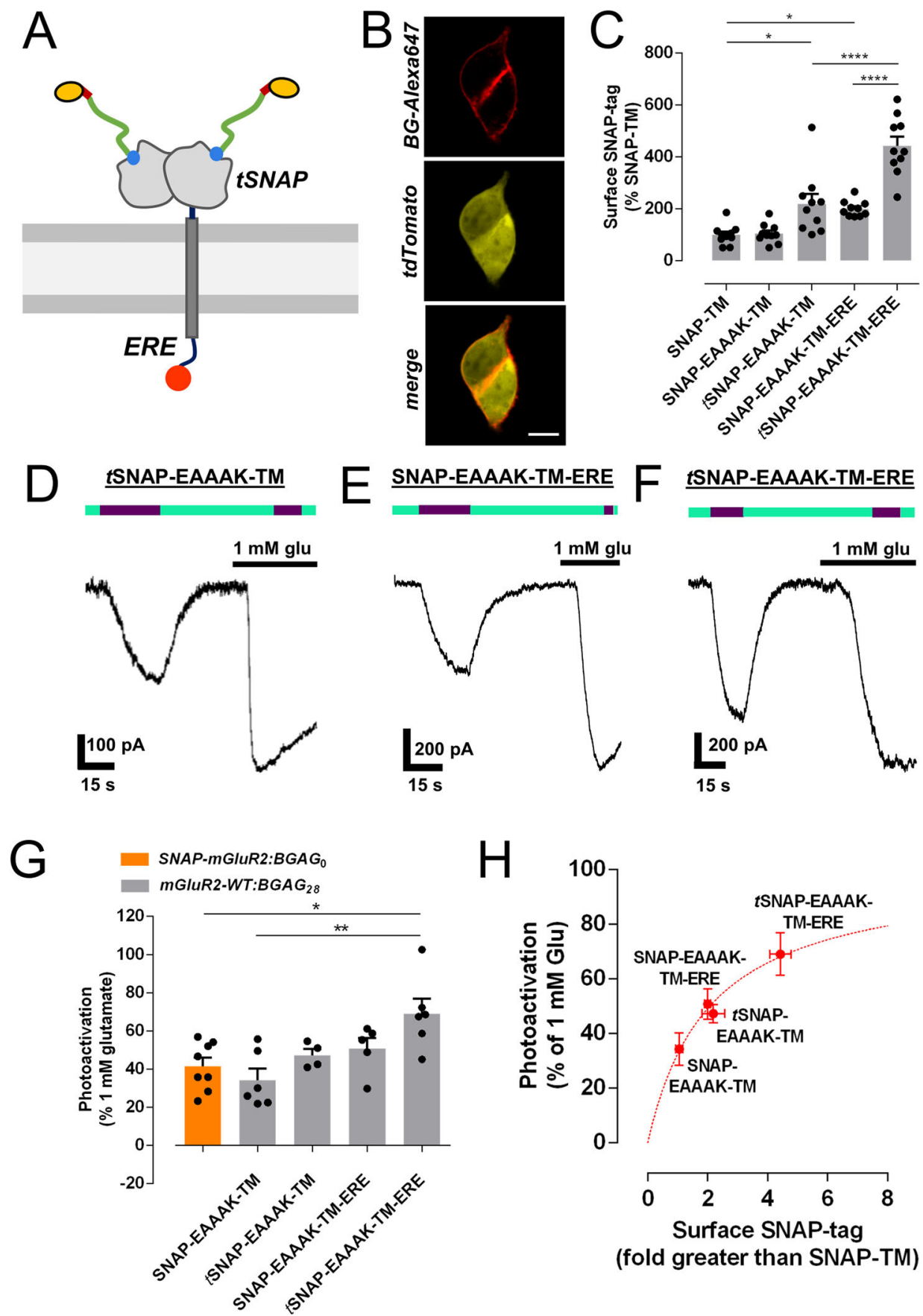

(fold greater than SNAP-TM)

Figure 5.

Increasing the number of membrane-anchored SNAP-tags enhances photoactivation of mGluR2-WT. (A) Schematic representation of two $\mathrm{BGAG}_{28}$ molecules bound to a SNAPTM variant with tandem SNAP-tags (ISNAP) as well as the endoplasmic reticulum export tag from Kir2.1 (ERE). (B) HEK293T cells expressing cytosolic tdTomato and tSNAPEAAAK-TM-ERE labeled with the membrane impermeant dye BG-Alexa647. scale bar = $10 \mu \mathrm{m}$. (C) Summary of surface SNAP-tag levels associated with variants of SNAP-TM (one-way ANOVA, Tukey). Each data point represents a field of many cells. (D-F) 
Representative traces of $\mathrm{BGAG}_{28}$-induced photoactivation of mGluR2-WT when tethered to (D) $t$ SNAP-EAAAK-TM, (E) SNAP-EAAAK-TM-ERE, and (F) tSNAP-EAAAK-TM-ERE.

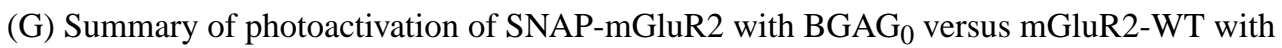
$\mathrm{BGAG}_{28}$ tethered to variants of SNAP-TM (one-way ANOVA, Tukey). (H) Photoactivation of mGluR2-WT increases with increasing levels of surface SNAP-tag. ${ }^{*} p<0.05, * * p<0.01$, $* * * * p<0.0001$. 

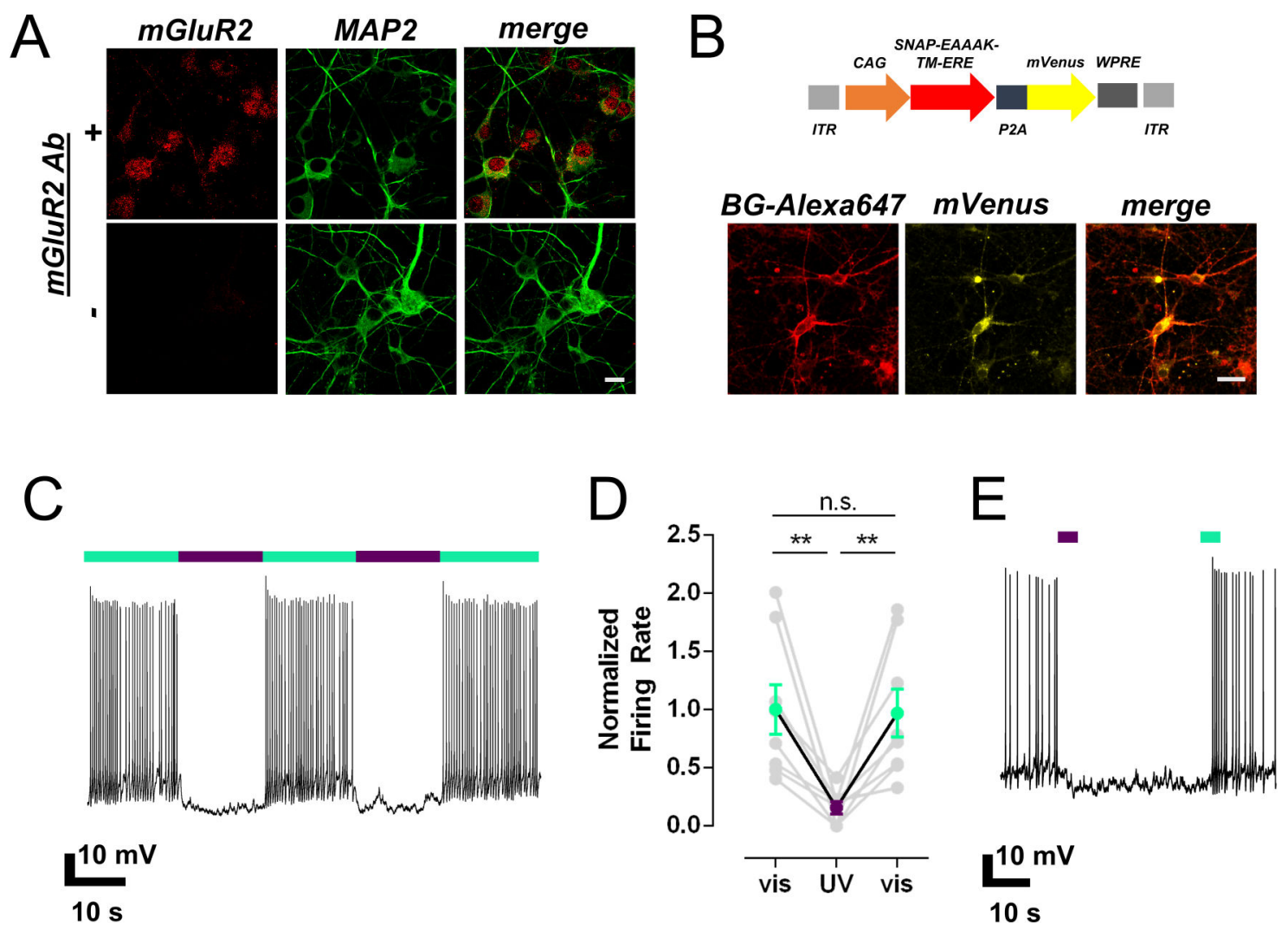

Figure 6.

SNAP-EAAAK-TM-ERE:BGAG 28 photoactivates endogenous $\mathrm{BGluR} 2_{2}$ in primary cortical neurons. (A) Rat primary cortical neurons (CNs), positive for the neuronal marker MAP2, express mGluR2 endogenously according to staining with an mGluR2 antibody (upper panels). Staining was not observed unless CNs were incubated with the mGluR2 antibody (lower panels). Scale bar $=15 \mu \mathrm{m}$. (B) Schematic of the expression cassette of an AAV encoding SNAP-EAAAK-TM-ERE (upper panel). CNs expressing BG-Alexa647 labeledSNAP-EAAAK-TM-ERE as well as the fluorescent reporter mVenus (lower panels). Scale bar $=30 \mu \mathrm{m}$. (C and D) Photoactivation of endogenous mGluR2 with SNAP-EAAAK-TMERE: $B_{A^{2}}$ results in a rapid, reversible, and repeatable suppression of spontaneous firing ( $n=8$, one-way ANOVA, Tukey). $* * p<0.01$. Firing was normalized to the average firing rate for all the neurons tested under the first pulse of visible light. Gray points and lines represent individual neurons. (E) The suppression of spontaneous firing was bistable. 